\title{
Identification of Nonlinear Time-lag System under Noisy Measurements Using Improved Genetic Algorithm
}

\author{
Toshiyuki ASAKURA* and $\bigcirc$ Qingzhi GUO* \\ *Faculty of Engineering, Fukui University, 3-9-1 Bunkyo, Fukui, Japan \\ asakura@scmc.mech.fukui-u.ac.jp
}

\begin{abstract}
In this paper, a new identification method is proposed which can obtain a good accuracy of identification of nonlinear time-lag systems under noisy measurements, on the basis of combination of genetic algorithm and sequence method. First, the identified system is described as a discrete model of a polynomial type with unknown parameters using Kolmogorov-Gabor's method. The task of system identification is to determine these parameters. Though the parameters of the identified system can be obtained through the search of GA, there is a potential risk in using a simple GA that a solution is usually stuck at a local minimum. Second, to solve this problem, a new GA search method is proposed by adding a sequence search which is carried out nearby the value of each estimated parameter coming from a simple GA. By this method, individuals whose fitness are larger are found. As a result, the solution escapes from the local minimum and converges to the optimum one. Finally, through simulation, some examples are given to demonstrate the validity of the proposed identification method.
\end{abstract}

\section{1 . Introduction}

In many model-based approaches, it is essential to build a good model. After the model structure is determined, the main task of the identification is to estimate model parameters. However, in a real nonlinear time-lag system, identification is very difficult ${ }^{1), 2}$. On the other hand, recent researches show that genetic algorithm(GA) is very powerful and has many advantages through its multi-point search with population ${ }^{3}, 4$ ). It can perform robust search, and has been applied in many fields ${ }^{5}$. Using the search of GA, an identification method has been proposed for nonlinear time-lag systems. ${ }^{6}$ ) But there is a potential risk that a simple GA search is usually stuck at a local minimum of loss function for a multimodal nonlinear time-lag system with noise, and thus results in a poorly identified model. To prevent this problem, a hybrid method will be proposed in this paper. This improved method can be summarized as below: the search can be carried out through all the space by GA and a good initial value is obtained, then the local search is conducted by sequence method, a better value is renewed progressively and identification accuracy is increased.

To examine the usefulness of the proposed method, several examples are given for nonlinear vibrating systems of Duffing and Van der pol type that composed of spring, damper with time-lag and noise. Through simulation studies, the effectiveness of the proposed method and the possibility of the identification of nonlinear time-lag system with noise are demonstrated .

\section{Problem Statement}

A general model of nonlinear time-lag system can be described as follows, ${ }^{7}$

$$
\begin{aligned}
\frac{d y(t)}{d t}= & f\left(y(t), y\left(t-h_{1}\right), \cdots, y\left(t-h_{y}\right),\right. \\
& \left.u(t), u\left(t-k_{1}\right), \cdots, u\left(t-k_{v}\right), \phi(t), t\right)
\end{aligned}
$$

where $y(t)$ is the output, $u(t)$ the input, $h_{i}(i=1,2 \cdots$, $\nu$ ) time-lag in system, $k_{j}(j=1,2, \cdots, v)$ time-lag of input, $\phi(\mathrm{t})$ the noise and $f$ nonlinear function. The nonlinear function can be expressed as a discrete model of polynomials with unknown coefficients by Kolmogorov-Gabor 's method.

$$
\begin{aligned}
& y(k)=f\left(V_{1}, V_{2}, \cdots, V_{s}\right)=\sum_{i=1}^{s} a_{i} V_{i} \\
& +\sum_{i=1}^{s} \sum_{j=1}^{s} b_{i j} V_{i} V_{j}+\cdots \\
& +\underbrace{\sum_{i=1}^{s} \sum_{j=1}^{s} \cdots \sum_{n=1}^{s} c_{i j \cdots n} V_{i} V_{j} \cdots V_{n}+\varepsilon(k)}_{m}
\end{aligned}
$$

where

$$
\begin{aligned}
& V_{1}=y(k-1), \cdots, V_{y+1}=y\left(k-1-d_{k y}\right), \\
& V_{y+2}=u(k-1), \cdots, V_{s}=u\left(k-1-d_{k v}\right), \\
& s=\gamma+v+2
\end{aligned}
$$

$\varepsilon(k)$ is the surplus part by finite terms. The estimated value of $y(k)$ is expressed as follows, 


$$
\begin{aligned}
\hat{y}(k) & =\sum_{i=1}^{s} \hat{a}_{i} V^{*}\left(\hat{d}_{k p}, \hat{d}_{k q}\right)+\sum_{i=1}^{s} \sum_{j=1}^{s} \hat{b}_{i j} V_{i}^{*}\left(\hat{d}_{k p}, \hat{d}_{k q}\right) V_{j}^{*}\left(\hat{d}_{k p}, \hat{d}_{k q}\right), \cdots, \\
& +\sum_{i=1}^{s} \sum_{j=1}^{s} \cdots \sum_{n=1}^{s} \hat{c}_{i j \cdots n} V_{i}^{*}\left(\hat{d}_{k p}, \hat{d}_{k q}\right) V_{j}^{*}\left(\hat{d}_{k p}, \hat{d}_{k q}\right) \cdots V_{n}^{*}\left(\hat{d}_{k p}, \hat{d}_{k q}\right)
\end{aligned}
$$

where $p=1,2 \cdots \gamma$ and $q=1,2 \cdots v$.

So the system identification is to determine the unknown parameters $\hat{a}_{\dot{b}} \hat{b}_{i j}, \cdots, \hat{c}_{i j \ldots n}, \hat{d}_{k p}$ and $\hat{d}_{k q}$.

\section{Identification Using the search of GA and Sequence method}

The identification using the proposed method based on the search of GA and sequence method can be described as two stages processes, in which first a good initial value is searched using GA, and then identification performed by the search of sequence method.

Genetic Algorithms which model on genetic processes occurring in nature, are adaptive methods that may be used to solve search and optimization problem. They work with a population of individuals (also known as chromosomes), which represent possible solutions to a given problem. Each individuals are assigned a fitness score according to how good a solution to the problem it is. The highly fit individuals are given opportunities to reproduce, by cross breeding with individuals in the population. Over several generations, individuals tend to identity and the solution is converged.

In this paper, the estimated parameters in Eq.(3) can be encoded as a chromosome which is a string of binary bits written as Eq.(6), and when the search is finished, the genetic information in binary numbers is returned to the parameters in decimal numbers by the transformation equation expressing in Eq.(4) and the estimated values of the parameters can be obtained.

$$
\begin{aligned}
& \hat{a}_{i}\left(\hat{b}_{i j,} \cdots, \hat{c}_{i j \cdots n}, \hat{d}_{k p}, \hat{d}_{k q}\right) \\
& \quad \hat{a}_{i}^{*}\left(\hat{b}_{i j}^{*} \cdots, \hat{c}_{i j \cdots n}^{*}, \hat{d}_{k p}^{*}, \hat{d}_{k q}^{*}\right) /\left(2^{p}-1\right)^{*} E_{k}
\end{aligned}
$$

where $E_{k}$ is the constant showing the upper limit of every estimated parameter.

The operating procedure of GA is given in figure 1 , where the individuals of the population in first generation may be given as random values. Then, the selection is done depending on the fitness of each individual. In this study, the fitness function is expressed as a loss function of rms error, and the smaller the rms error the better.

$$
J=\sqrt{\frac{1}{z} \sum_{k=1}^{z}(y(k)-\hat{y}(k))^{2}}
$$

where $z$ is the number of data.

Selection is that the individuals with small rms error can survive and the poor ones with big rms error will be culled. Crossover is applied to the pairs of survived individuals to regenerate the culled ones,

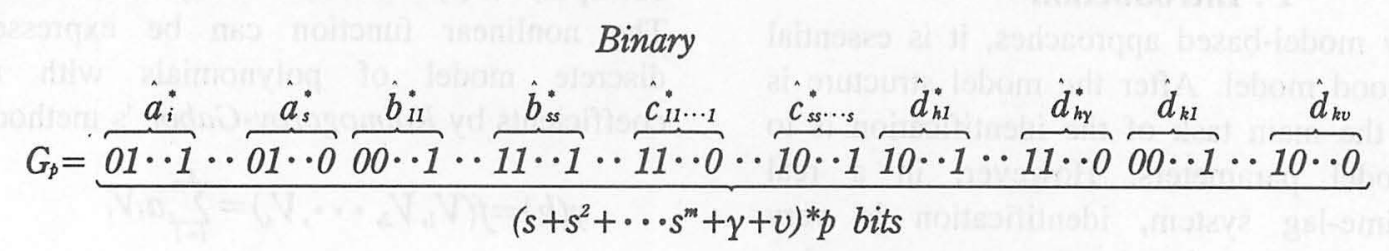

The Nth generation

The $(\mathrm{N}+1)$ th generation

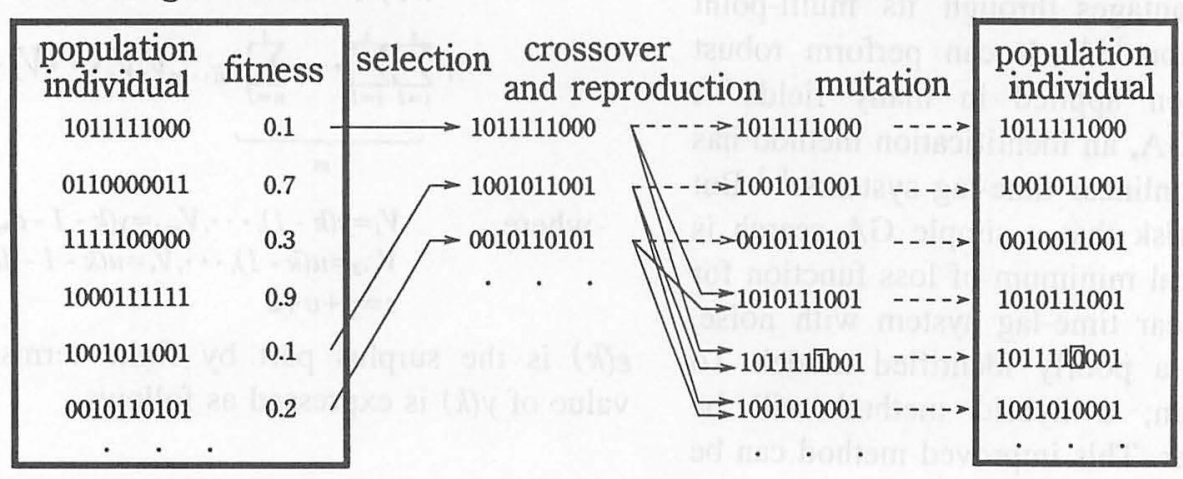

Fig.1 GA operation 


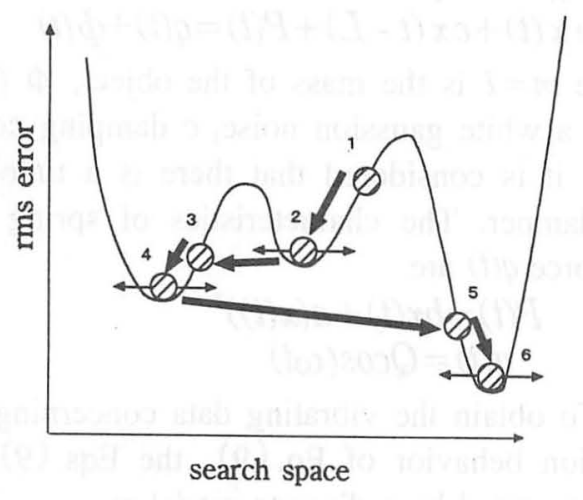

Fig.2 Sequence method

through replacing the bits of chromosomes at random choice. Moreover, mutation is used to the new progenies and every gene is altered randomly with a probability. A good initial value can be obtained by repeating such a kind of evolution. However, it should be noticed that when a simple GA is used to multimodal nonlinear systems, it is easy to be blocked at a local minimum and the solution at this point will be much different from the finally close one.

To solve this problem, the sequence method is introduced for local search to escape from the local minimum by renewing the initial value. This approach is shown in Fig.2: in the first, the rms error of individuals generated at random is on the position 1, and through the process of GA search, the rms error is converged to position 2, a local minimum solution. To escape from this, the sequence search is carried out subsequently. By adjusting the values of parameters finely, individuals whose fitness are larger are generated. As a result, the solution escapes from the local minimum and converges to the optimum one whose rms error is on position 3. After escaping from the local minimum, the search is returned to GA. Then, the search is done again and again.

$1 \rightarrow 2 \longrightarrow 3 \longrightarrow 4 \longrightarrow 5 \rightarrow 6$

$G A \Rightarrow$ Sequence search $\Rightarrow G A \Rightarrow$ Sequence search $\Rightarrow \cdots$

The renewal of the parameters with sequence method is expressed as follows,

$$
\begin{aligned}
& \hat{a}_{i}\left(\hat{b}_{i j}, \cdots, \hat{c}_{i j \cdots n}, \hat{d}_{h p}, \hat{d}_{k q}\right)_{\text {times } \mathrm{T}+1} \\
& \quad=\hat{a}_{i}\left(\hat{b}_{i j}, \cdots, \hat{c}_{i j \cdots n}, \hat{d}_{h p}, \hat{d}_{k q}\right)_{\text {times } T} \pm \beta_{k} \\
& \beta_{k}=\delta^{*} \hat{a}_{i}\left(\hat{b}_{i j}, \cdots, \hat{c}_{i j \cdots n}, \hat{d}_{h p}, \hat{d}_{k q}\right)_{\text {times T }}
\end{aligned}
$$

where, $\hat{a}_{i}\left(\hat{b}_{i j}, \cdots, \hat{c}_{i j \cdots, n}, \hat{d}_{k p}, \hat{d}_{k q}\right)_{\text {times } T}$ is the estimated parameters of the $T$ th generation,

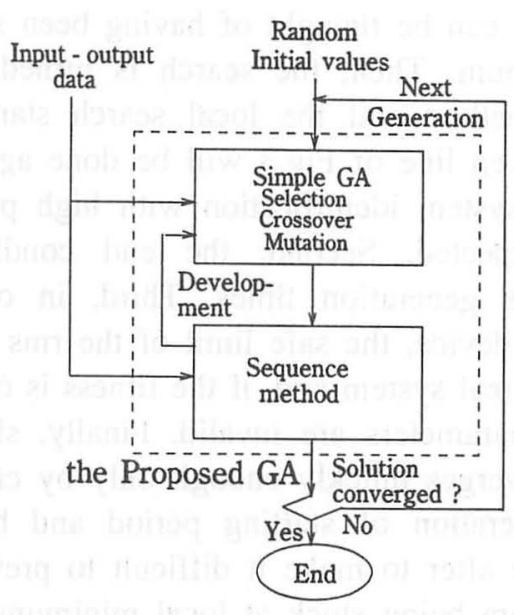

Fig.3 Image of improved GA

$\hat{a}_{i}\left(\hat{b}_{i}, \cdots, \hat{c}_{i j \cdots, \ldots}, \hat{d}_{k p}, \hat{d}_{k q}\right)_{\text {times } T+1}$ the ones of the $(T+1)$ th generation, $\beta_{k}$ the coefficient of sequence search, which determined by Eq.(8) and $\delta$ a small positive value.

It is supposed that the parameters, obtained by GA at the $T$ th generation times, are

$$
\begin{aligned}
& \hat{a}_{T 1}, \cdots, \hat{a}_{T}, \cdots, \hat{a}_{T s}, \hat{b}_{T l 1}, \cdots, \hat{b}_{T i j}, \cdots \hat{b}_{T s s}, \\
& \quad \cdots, \hat{c}_{T l 1 \cdots 1}, \cdots, \hat{c}_{T i j \cdots n}, \cdots, \hat{c}_{T s \cdots s}, \\
& \hat{d}_{T h 1}, \cdots, \hat{d}_{T h p}, \cdots, \hat{d}_{T h \mathrm{y}}, \hat{d}_{T k l}, \cdots, \hat{d}_{T k q}, \cdots, \hat{d}_{T h v}
\end{aligned}
$$

Depending on Eq.(7), those of the $(T+1)$ th generation times can be obtained as follows,

$$
\begin{aligned}
& \hat{a}_{T l}, \cdots,\left(\hat{a}_{T 1}+\beta_{i}\right), \cdots, \hat{a}_{T s}, \hat{b}_{T 11}, \cdots, \hat{b}_{T i j}, \cdots \hat{b}_{T s s}, \\
& \cdots, \hat{c}_{T I \ldots 1}, \cdots,\left(\hat{c}_{T i j \cdots n}-\beta_{i j \ldots n}\right), \cdots, \hat{c}_{T s, \cdots s}, \\
& \hat{d}_{T h l}, \cdots, \hat{d}_{T h p}, \cdots, \hat{d}_{T h \mathrm{Y}}, \hat{d}_{T k l}, \cdots, \hat{d}_{T k q}, \cdots, \hat{d}_{T h v} \\
& \hat{a}_{T 1}, \cdots, \hat{a}_{T 1}, \cdots, \hat{a}_{T s}, \hat{b}_{T 11}, \cdots,\left(\hat{b}_{T i j}-\beta_{i j}\right), \cdots \hat{b}_{T s}, \\
& \cdots, \hat{c}_{T 11 \ldots 1}, \cdots,\left(\hat{c}_{T i j \cdots n}+\beta_{i j \cdots n}\right), \cdots, \hat{c}_{T s, \cdots s}, \\
& \hat{d}_{T h l}, \cdots,\left(\hat{d}_{T h p}+\beta_{h p}\right), \cdots, \hat{d}_{T h Y}, \hat{d}_{T k 1}, \cdots, \hat{d}_{T k q}, \cdots, \hat{d}_{T k v} \\
& \hat{a}_{T 1}, \cdots,\left(\hat{a}_{T-}-\beta_{i}\right), \cdots, \hat{a}_{T s}, \hat{b}_{T 11}, \cdots, \hat{b}_{T i j}, \cdots \hat{b}_{T s}, \\
& \cdots, \hat{c}_{T 11 \cdots 1}, \cdots,\left(\hat{c}_{T i j \cdots n}-\beta_{i j \cdots n}\right), \cdots, \hat{c}_{T s, \ldots s}, \\
& \hat{d}_{T h 1}, \cdots, \hat{d}_{T h p}, \cdots, \hat{d}_{T h \mathrm{\gamma}}, \hat{d}_{T k 1}, \cdots,\left(\hat{d}_{T k q}+\beta_{k q}\right), \cdots, \hat{d}_{T k \mathrm{v}}
\end{aligned}
$$

By introducing a new GA operator named as development, we propose an improved GA, which is an effective combination of genetic algorithm and sequence method. A hybrid identification algorithm based on this consideration is shown in Fig.3. Also, the principle of this improved GA is very easy, but there are some important points in realizing it. First, 
if the fitness has no change in 30 generation times, the solution can be thought of having been stuck at local minimum. Then, the search is turned to the sequence method and the local search starts. The part in broken line of Fig. 3 will be done again and again, the system identification with high precision can be expected. Second, the end condition is designed as generation times. Third, in order to protect the device, the safe limit of the rms error is set up in a real system and, if the fitness is over this limit, the parameters are invalid. Finally, since the fitness converges quickly enough only by crossover in the generation of starting period and becomes slow in the after to make it difficult to prevent the solution from being stuck at local minimum. So, in this study, the mutation rate is made small in the starting period and large then.

\section{Simulation Studies}

Numerical simulations to the systems of Duffing, Piece wise and Van der pol type have been performed to demonstrate the effectiveness of the proposed identification method. It's supposed each of the systems includes time-lag and the results are compared with those using the search of simple GA. Each parameters used in GA are set as,

\section{Total of individuals $=50$ \\ Selection rate $=50 \%$ \\ Mutation rate $=20 \%, 80 \%$ \\ Generation times $=400$ \\ Data numbers $=300$}

The mutation rate in GA operation is $20 \%$ in the first 50 generations and $80 \%$ subsequently. The renewed coefficient in sequence method is supposed as

$$
\beta_{\mathrm{k}}=0.01
$$

for the sake of convenience.

\subsection{Duffing type nonlinear time-lag system}

As the system model, a nonlinear time-lag system of Duffing type is considered whose dynamics is written as
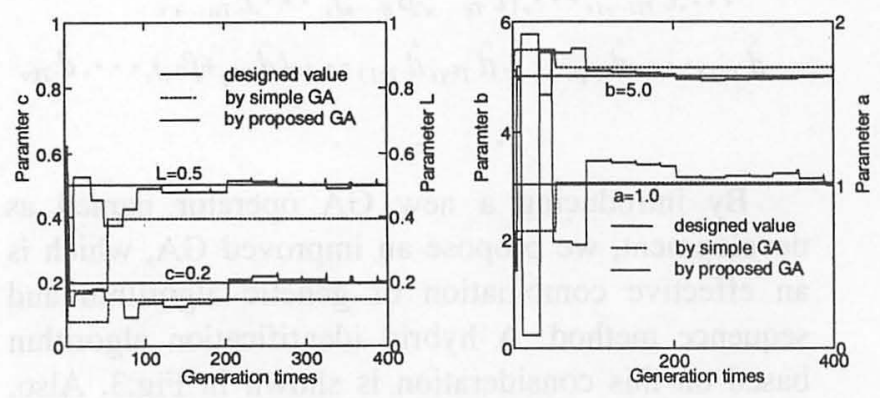

Fig. 4 Estimated parameters c, L, b, a

$$
m \ddot{x}(t)+c \dot{x}(t-L)+P(t)=q(t)+\phi(t)
$$

where $m=1$ is the mass of the object, $\phi(t) \in N(0$, 0.01 ) a white gaussion noise, $c$ damping coefficient. Here, it is considered that there is a time-lag $L$ in the damper. The characteristics of spring $P(t)$ and the force $q(t)$ are

$$
\begin{gathered}
P(t)=b x(t)+a(x(t))^{3} \\
q(t)=Q \cos (\omega t)
\end{gathered}
$$

To obtain the vibrating data concerning with the solution behavior of Eq. (9), the Eqs. (9) (11) are expressed by a discrete model as ,

$$
\begin{aligned}
x(k+2)= & 2 x(k+1)-\lambda_{1}(x(k+1-d)-x(k-d)) \\
& -\lambda_{2} x(k)-\lambda_{3}(x(k))^{3}+\lambda_{4} \cos (\omega k)+\phi(t)
\end{aligned}
$$

where

$$
\begin{array}{ll}
\lambda_{1}=c h / m, & \lambda_{2}=1+h^{2} b / m \\
\lambda_{3}=h^{2} a / m, & \lambda_{4}=h^{2} Q / m, \quad d=L / h
\end{array}
$$

The parameters of Eq.(12) are set as

$$
c=0.2, L=0.5, b=5.0, a=1.0 \text {. }
$$

The input parameters are $Q=10.0, \omega=5.0$, and sampling period of time $h=0.02 \mathrm{sec}$.

Table 1 shows the identified result. The datum file (1) is the true values of the parameters, file (2) the identified results using the search of simple GA

\begin{tabular}{|c|c|c|c|c|}
\hline \multirow{2}{*}{$\begin{array}{c}\text { Reference } \\
\text { value } \\
\text { (1) }\end{array}$} & \multicolumn{4}{|c|}{ Estimated parameters } \\
\hline & $\begin{array}{c}\text { Simple GA } \\
\text { (2) }\end{array}$ & $\begin{array}{l}\text { Error } \\
\text { |(1)-(2) | }\end{array}$ & $\begin{array}{l}\text { Proposed GA } \\
\text { (3) }\end{array}$ & $\begin{array}{l}\text { Error } \\
\mid \text { (1)-(3) } \mid\end{array}$ \\
\hline$c=0.2$ & 0.143109 & 0.0569 & 0.195112 & 0.0049 \\
\hline $\mathrm{L}=0.5$ & 93939 & 0.1061 & 7556 & 0.0024 \\
\hline$b=5.0$ & 5.171065 & 0.1711 & 5.004888 & 0.0049 \\
\hline$a=1.0$ & 1.018573 & 0.0186 & 1.012708 & 0.0127 \\
\hline
\end{tabular}
and (3) those of improved GA. As shown in Table 1, the identification accuracy of each parameters is higher in the case of proposed GA. Fig. 4 shows the converging processes of each parameters. Also, Fig. 5 shows the output response and identified results of the object and rms error, respectively. It can be seen from Fig.5 that the solution sank into local minimum

Table 1 Result of estimated parameters
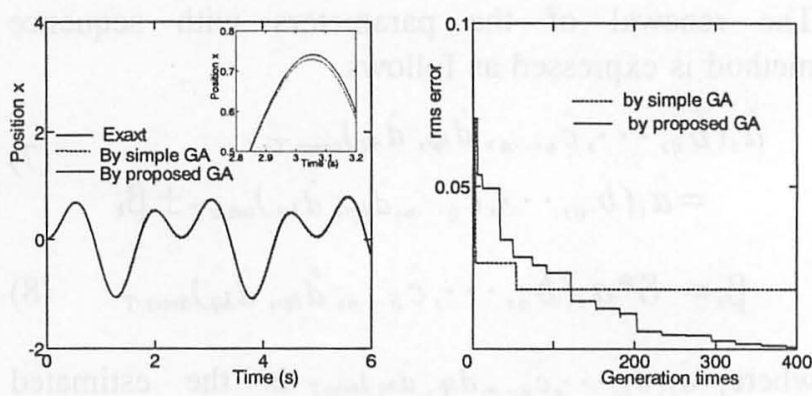

Fig.5 Output responses and rms error 
Table 2 Result of estimated parameters

\begin{tabular}{|c|c|c|c|c|}
\hline \multirow{2}{*}{$\begin{array}{c}\text { Reference } \\
\text { value } \\
(1)\end{array}$} & \multicolumn{4}{|c|}{ Estimated parameters } \\
\cline { 2 - 5 } & $\begin{array}{c}\text { Simple GA } \\
\text { (2) }\end{array}$ & $\begin{array}{c}\text { Error } \\
\text { (1)-(2) }\end{array}$ & $\begin{array}{c}\text { Proposed GA } \\
(3)\end{array}$ & $\begin{array}{c}\text { Error } \\
\text { | (1)-(3) I }\end{array}$ \\
\hline $\mathrm{c}=0.2$ & 0.233822 & 0.0382 & 0.215445 & 0.0154 \\
\hline $\mathrm{L}=0.5$ & 0.532747 & 0.0327 & 0.517107 & 0.0171 \\
\hline $\mathrm{b}=5.0$ & 4.877810 & 0.1222 & 4.956012 & 0.0440 \\
\hline$\mu=0.5$ & 0.466276 & 0.0337 & 0.520039 & 0.0200 \\
\hline
\end{tabular}
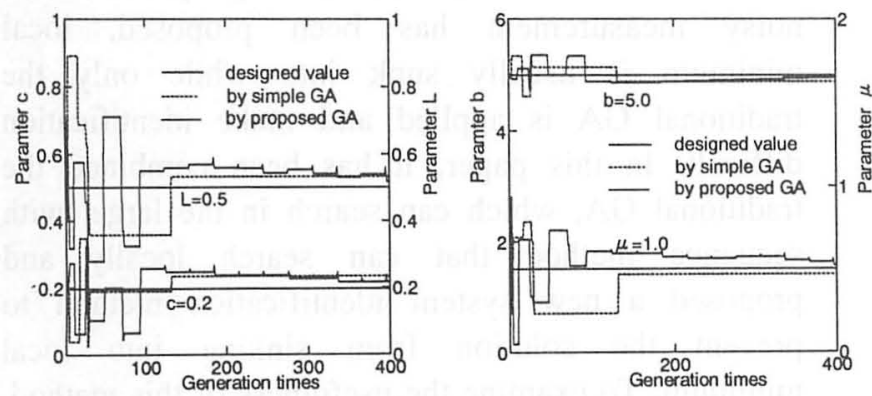

Fig. 6 Estimated parameters c, L, b, $\mu$
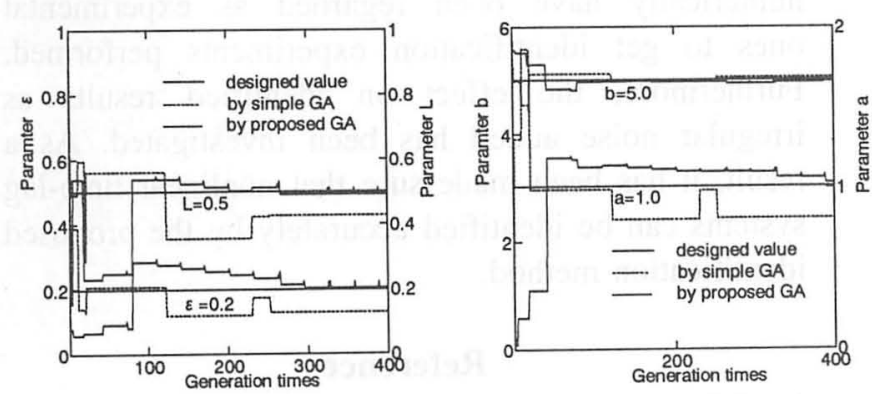

Fig. 8 Estimated parameters c, L, b, $\varepsilon$

at about 54th generation point, when searching by simple GA, and the parameters stopped converging. Then, it's hard to increase identification accuracy any longer. On the other hand, in the searching by proposed GA, local minimum will be sunk into as above-mentioned, if rms error has not been improved at every 30 generations and while sequence method works, the solution can escape from the local minimum. As a result, the estimated parameters get closer and closer to true ones and accord with them and the identification with high accuracy has been performed.

The real systems with the existence of piece wise such as a coulomb friction, are not always smoothly nonlinear. As an example, this one has been selected to be identified. The system model is shown in Eq. (9) but the nonlinear term is

$$
P(t)=b x(t)+\mu \operatorname{sgn}(\dot{x})
$$

where $\mu$ is the coulomb friction coefficient.
Table 3 Result of estimated parameters

\begin{tabular}{|c|c|c|c|c|}
\hline \multirow{2}{*}{$\begin{array}{c}\text { Reference } \\
\text { value } \\
\text { (1) }\end{array}$} & \multicolumn{4}{|c|}{ Estimated parameters } \\
\cline { 2 - 5 } & $\begin{array}{c}\text { Simple GA } \\
\text { (2) }\end{array}$ & $\begin{array}{c}\text { Error } \\
\text { (1)-(2) }\end{array}$ & $\begin{array}{c}\text { Proposed GA } \\
\text { (3) }\end{array}$ & $\begin{array}{c}\text { Error } \\
\text { (1)-(3) । }\end{array}$ \\
\hline$\varepsilon=0.2$ & 0.127859 & 0.0724 & 0.201369 & 0.0014 \\
\hline $\mathrm{L}=0.5$ & 0.426197 & 0.0738 & 0.490714 & 0.0093 \\
\hline $\mathrm{b}=5.0$ & 5.063539 & 0.0635 & 4.985337 & 0.0147 \\
\hline $\mathrm{a}=1.0$ & 0.809384 & 0.1906 & 1.030303 & 0.0303 \\
\hline
\end{tabular}
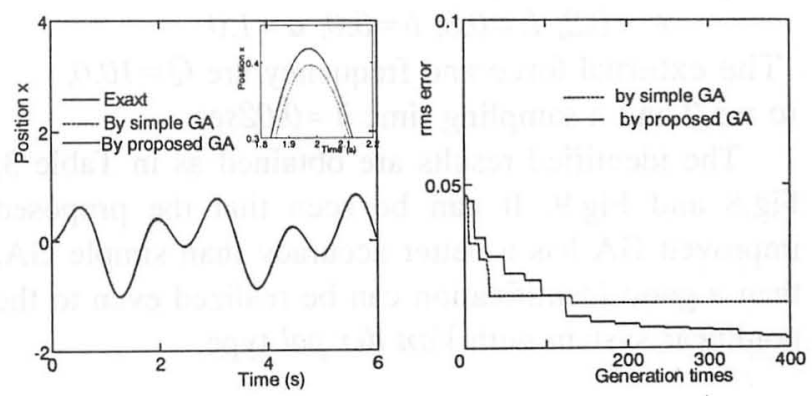

Fig.7 Output responses and rms error
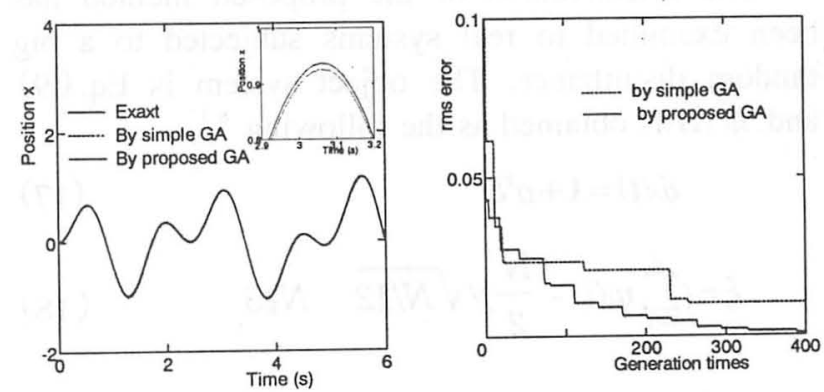

Fig.9 Output responses and rms error

$$
\operatorname{sgn}(\dot{x})= \begin{cases}1 & (\dot{x}>0) \\ -1 & (\dot{x}<0)\end{cases}
$$

The parameters are given as

$$
c=0.2, L=0.5, b=5.0, \mu=1.0
$$

The identified results are illustrated in Table 2, Fig.6 and Fig.7, as the same as those of above mentioned. From these it can be known that the proposed identification method can identify even piece wise type system with a good accuracy.

\subsection{Van der pol type nonlinear time-lag system}

The characteristics of damper is given by Van der pol type equation with time-lag. The dynamic equation is written as

$$
m \ddot{x}-\varepsilon\left(1-x^{2}\right) \dot{x}(t-L)+b x+a x^{3}=Q \cos (\omega t)+\phi(t)
$$

The discrete difference equation is derived as follows, 


$$
\begin{aligned}
x(k+2)= & 2 x(k+1)+\lambda_{5}(x(k+1-d)-x(k-d)) \\
& -\lambda_{\sigma}\left(x(k)^{2}(x(k+1-d)-x(k-d))\right) \\
& -\lambda_{7} x(k)-\lambda_{8}(x(k))^{3}+\lambda_{9} \cos (\omega k)+\Phi(t)
\end{aligned}
$$

where

$$
\begin{aligned}
& \lambda_{5}=\lambda_{6}=\varepsilon h / m, \quad \lambda_{7}=1+h^{2} b / m \\
& \lambda_{8}=h^{2} a / m, \quad \lambda_{9}=h^{2} Q / m, \quad d=L / h
\end{aligned}
$$

The parameters are given as

$$
\varepsilon=0.2, L=0.5, b=5.0, a=1.0
$$

The external force and frequency are $Q=10.0$, $\omega=5.0$ and a sampling time $h=0.02 \mathrm{sec}$.

The identified results are obtained as in Table 3, Fig.8 and Fig.9. It can be seen that the proposed improved GA has a better accuracy than simple GA, then a good identification can be realized even to the nonlinear system with Van der pol type.

\subsection{The effect of large noise}

\section{on the identification accuracy}

The effectiveness of the proposed method has been examined to real systems subjected to a big random disturbance. The object system is Eq. (9) and $\phi(t)$ is obtained as the following ${ }^{8}$ ),

$$
\begin{gathered}
\phi(t)=\lambda+\sigma^{2} \xi \\
\xi=\left(\sum_{i=1}^{N} \psi(i)-\frac{N}{2}\right) / \sqrt{N / 12} \quad N \geqq 3
\end{gathered}
$$

In Eq. $(18), \psi(t)$ is determined randomly to the range of $-1.0 \sim 1.0$. In Eq. (17) the mean value $\lambda$ is zero.

The parameters are set as the same of those in section 4.1. In order to examine the identification accuracy, simulations of the relation between noise and rms error are performed. Fig.10 gives the result, where the round marks indicate experimental values. It can be seen from these results that

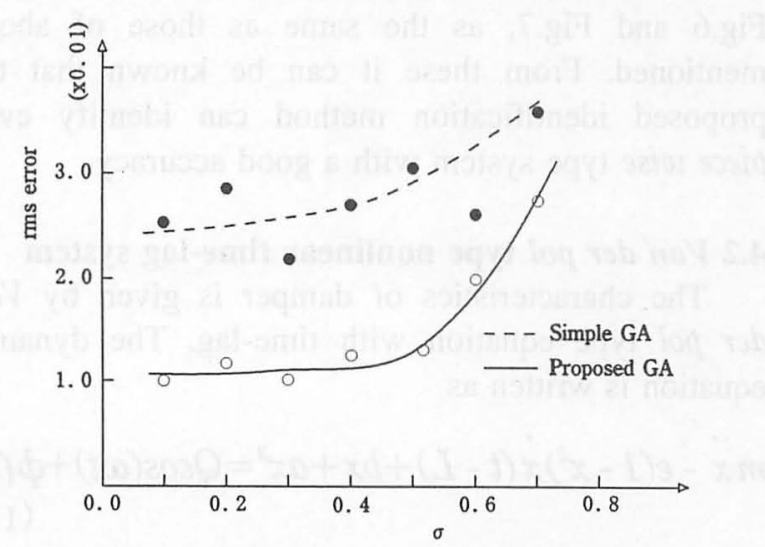

Fig.10 Relation between noise and rms error identification accuracy can be improved even noise is large. However, larger the noise larger the rms error. The rms error will become large acutely as the standard deviation of noise turns to $0.5(\mathrm{~S} / \mathrm{N}=14.14)$ over and identification will become worse even if the proposed improved GA is used.

\section{5 . Conclusions}

Though an identification method using the search of GA to nonlinear time-lag system under noisy measurement has been proposed, local minimum is usually sunk into while only the traditional GA is applied and make identification difficult. In this paper, it has been combined the traditional GA, which can search in the large, with sequence method that can search locally and proposed a new system identification method to prevent the solution from sinking into local minimum. To examine the usefulness of this method, several representative nonlinear time-lag systems have been selected and the vibrating data obtained numerically have been regarded as experimental ones to get identification experiments performed. Furthermore, the effect on identified results as irregular noise added has been investigated. As a result, it has been made sure that nonlinear time-lag systems can be identified accurately by the proposed identification method.

\section{Reference}

1) S.Sagara, K.Akitsuki, T.Nakamizo, T.Katayama, System identification, p3, the society of instrument and control engineers, Japan (1980)

2) X.Adati, System identification theory, p200, the society of instrument and control engineers, Japan (1983)

3) D.E.Goldberg, Genetic Algorithms in Search Optimization, and Machine Learning, Addison-Wesley (1989)

4) M.Sakawa, M.Tanaka, Genetic algorithm, Asakura books, p178

5) K.Iizuka, Measurement and control, Vol.32 No.1 Jan. 1993 39/62

6) Q.Z. Guo, T. Asakura, Identification of Nonlinear time lag System by Genetic Algorithm, The 39th Japan Joint Automatic Control Conference 157/158

7) A.Ichigawa, Automatic Control Handbook, p764, the society of instrument and control engineers, Japan (1983)

8) Y. Takahashi, Digital Control, (1985) p203 Iwaha books 